\title{
A Low-Complexity Resource Allocation Algorithm for Multicast Service Delivery in OFDMA Networks
}

\author{
G. Araniti, M. Condoluci, A. Iera, A. Molinaro, J. Cosmas, M. Behjati
}

\begin{abstract}
Allocating and managing radio resources to multicast transmissions in Orthogonal Frequency-Division Multiple Access (OFDMA) systems is the challenging research issue addressed by this paper. A subgrouping technique, which divides the subscribers into subgroups according to the experienced channel quality, is considered to overcome the throughput limitations of conventional multicast data delivery schemes. A low complexity algorithm, designed to work with different resource allocation strategies, is also proposed to reduce the computational complexity of the subgroup formation problem. Simulation results, carried out by considering the Long Term Evolution (LTE) system based on OFDMA, testify the effectiveness of the proposed solution, which achieves a near-optimal performance with a limited computational load for the system.
\end{abstract}

Index Terms-OFDMA, RRM, Frequency scheduling, Multicast, Subgrouping, LTE

\section{INTRODUCTION}

$\mathbf{I}$ $\mathrm{N}$ the current scenario of very fast web service expansion, high quality group-oriented (i.e., multicast and broadcast) services such as multimedia downloading, video conferencing, and mobile TV are gaining in importance. The design of effective multicast traffic delivery strategies in the Orthogonal Frequency-Division Multiple Access (OFDMA)based systems has become a challenging task investigated in several research works. Thanks to the great flexibility in spectrum management and the high robustness against fading phenomena, OFDMA is the basis of the most promising radio access systems, such as Long Term Evolution (LTE) [1] and Worldwide Interoperability for Microwave Access (WiMAX) [2], which support the transmissions of multicast contents in addition to the traditional unicast [3] [24].

In these systems, the design of efficient and effective Radio Resource Management (RRM) policies is essential to guarantee high-quality multicast sessions. Specifically, a key role is played by the link adaptation procedure, which selects the transmission parameters for each scheduling resource on a per-group basis, i.e., according to the channel conditions of all multicast users. The conventional multicast scheme [5] shows low efficiency since it assigns the group data rate based on the user experiencing the worst channel quality. To overcome this constraint, multi-rate approaches [4] have been proposed. Among them, subgroup-based policies [11] [12]

G. Araniti, M. Condoluci, A. Iera and A. Molinaro are with the DIIES Dep., University Mediterranea of Reggio Calabria, Italy, e-mail: \{araniti, massimo.condoluci, antonio.iera, antonella.molinaro\}@unirc.it.

J. Cosmas and M. Behjati are with the WNCC, Brunel University London, UK, e-mail: \{john.cosmas, mohammadreza.behjati\}@brunel.ac.uk. have been developed to improve the spectral efficiency and the session performance of multicast transmissions in OFDMA systems. A possible scenario is depicted in Fig. 1. Behind this approach there is the idea of dividing the multicast receivers into different subgroups according to the experienced channel conditions, and assigning them the transmission resources accordingly.

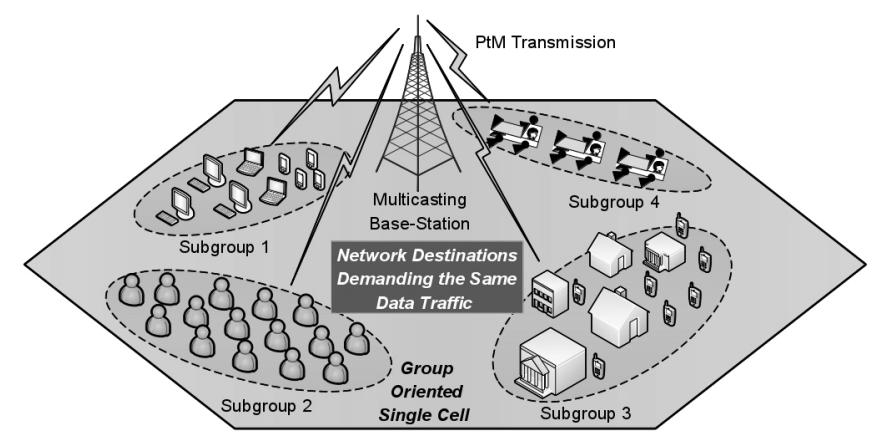

Fig. 1. The Multicast Sub-grouping Technique.

Subgrouping can noticeably improve the multicast session performance, although it can be characterized by a higher computation complexity than conventional schemes. Indeed, the need for selecting an adequate number of subgroups, with their relevant transmission parameters and assigned resources, introduces complexity in the subgroup creation. This makes an exhaustive search-based approach not suitable for real implementations. The design of an effective near-optimal policy for subgroup formation in OFDMA-based networks is still an open issue [13]. In this paper we propose a lowcomplexity greedy algorithm based on an iterative smart search of the most suitable subgroup configuration according to the channel qualities of users involved in the session. The proposed subgroup formation strategy is designed to work with different target cost functions. Through simulations, conducted by varying the channel bandwidth and multicast group size, we demonstrate the effectiveness of the proposed policy, which offers a better near-optimal performance and less iterations for convergence compared to the results achieved by the existing approaches in the literature.

The remainder of the paper is organized as follows. Section II provides a brief state of the art on link adaptation approaches for multicast content delivery. In Section III the addressed resource allocation problem is introduced, whereas Section IV focuses on the proposed low-complexity algorithm for 
subgroup formation in OFDMA-based systems. Section V provides the results achieved by simulation campaigns, while conclusion and future works are summarized in Section VI.

\section{RELATED WORK}

This paper focuses on single-cell multicast services, in which the base station delivers the data traffic to multiple receivers in the same multicast group. A group-oriented environment is characterized by the presence of multiple destinations requiring the same data traffic. In this environment, point-to-multipoint transmissions exploit the broadcast nature of the radio channel by using a single transmission to feed the whole multicast group. Multicasting over OFDMA-based networks introduces several open research issues, mainly due to the selection of the modulation and coding scheme (MCS), which is performed on a per-group basis.

Two main strategies for multicast data delivery have been proposed: single-rate and multi-rate schemes [4]. According to the former technique, all the group members are served with the same data rate. The Conventional Multicast Scheme (CMS) [5] belongs to this category. Although the CMS maximizes the system coverage (i.e., the number of destinations successfully served), this policy adopts a conservative approach by selecting the MCS according to the group member with the worst channel quality. As a consequence, the OFDMA potential is not fully exploited, and this negative aspect is more evident as the group size increases [6].

Another solution for single-rate scheduling policies is the opportunistic approach [7], which foresees, during any given time slot, to serve only the "best" portion of multicast members to maximize the Quality of Service (QoS) of the served users. Works in [8] and [9] propose threshold-based RRM policies. According to the approach in [8], terminals experiencing a SINR value lower than a threshold are not considered in scheduling decisions, whereas in [9] the MCS for the multicast transmission is chosen with the aim to achieve a target spectral efficiency of $1 \mathrm{bit} / \mathrm{s} / \mathrm{Hz}$. In [10], authors propose different opportunistic multicast scheduling algorithms to maximize the total throughput and to analyze the throughput and delay tradeoffs for each considered algorithm. Although the approaches proposed in [8]-[10] allow meaningful throughput improvements, the price to pay for such improvements is a reduction in the number of users served in each time slot. Moreover, opportunistic multicasting requires the use of rateless coding for guaranteeing the reception of data streams to the users served in different time slots [7].

The multi-rate multicast schemes can overcome the limitations of the CMS and opportunistic techniques by taking advantage from the intrinsic heterogeneity of the channel quality experienced by the multicast group members and by exploiting Hierarchical Layering (HL) or Multiple Description Coding (MDC) techniques [4]. In order to make the best of the radio channel capacity and the multi-user diversity, the multirate schemes divide the multicast group in smaller subgroups, based on intra-subgroup users' channel conditions. In such a context, the base station has to compute the most adequate number of subgroups to activate, and to select the MCS and the number of resources to assign to each enabled subgroup. Authors in [11] and [12] demonstrated that a subgroupbased resource allocation can significantly improve the performance in multicast content delivery over LTE networks. From these works it clearly emerges that subgrouping policies in OFDMA-based networks pose additional constraints in terms of computation complexity and this asks for the design of low-complexity algorithms for multicast subgroup formation. Focusing on this aspect, works in [13] and [22] dealt with a near-optimal subgroup formation for maximizing the sum of the data rate experienced by the multicast users, i.e., the Aggregate Data Rate (ADR). In particular, authors in [13] proposed the Subgroup Merging Scheme (SMS) that, in the initialization step, serves the multicast users on unicast connections with a random sub-carriers assignment. Once the initial cost function value is computed, the base station searches through all combinations of two subgroups to merge and selects the combination that guarantees the highest ADR increase. This process is iterated until there is no further ADR improvement or no subgroups to merge. Authors in [22] designed an efficient scheme, namely the Multicast Grouping Genetic Algorithm (MGGA), an evolutionary clustering method where the subgrouping problem is coded as subgroups in chromosomes and as fitness in ADR. The MGGA aims at performing different generations (i.e., iterations) in order to select the population (i.e., subgroup configuration) with the highest fitness (i.e., ADR). The SMS and MGGA schemes are suitable for practical implementations, although their effectiveness compared to other low-complexity approaches still needs to be evaluated. In this work we extend the referred works by (i) designing a general framework for subgroup formation in OFDMA-based networks which can be properly tuned to work with different target cost functions, and (ii) proposing a low complexity algorithm, which aims at (iii) guaranteeing a system performance close to the one achieved by an exhaustive search approach.

\section{SUBGROUP BASED RRM ALGORITHM}

\section{A. System model}

In this work we refer to a single-cell OFDMA system where a base station serves a single multicast group over a channel bandwidth equal to $B$. The station runs the RRM procedures by managing the available frequency resources within a given scheduling frame. Each scheduling resource corresponds to the smallest frequency unit managed by the RRM, e.g., a single sub-carrier or a subchannel composed of several adjacent subcarriers. The latter solution is implemented in many OFDMAbased systems, such as WiMAX [2] and LTE [1]. Let $R$ be the number of scheduling resources available for the multicast session and let $B_{o}=B / R$ be the channel bandwidth of each frequency resource.

The base station relies on the Channel State Information (CSI) forwarded by the multicast users every scheduling frame to decide the resource assignment. The CSI feedback is an indication of the channel quality of a given terminal and depends on the measured Signal to Interference plus Noise Ratio (SINR). Based on the gathered CSI information, the base 
station selects the most suitable MCS for each received CSI. Let $M$ be the number of the available MCS levels, and $c_{m}$ (with $m=1, \ldots, M$ ) the spectral efficiency (i.e, the number of transmitted data bits per Hertz) of the generic $m$-th modulation and coding scheme. The higher the MCS level, the higher the spectral efficiency, i.e., $c_{m^{*}}>c_{m}$, with $m^{*}>m$. Obviously, $c_{m} B_{o}$ represents the data rate achieved by one frequency resource when it is transmitted with the $m$-th MCS level.

Let $\mathcal{K}$ be the multicast user set, being $K=|\mathcal{K}|$ the multicast group size. We identify with $C S I_{k}(k=1, \ldots, K)$ the CSI feedback from the $k$-th user. Without loss of generality, we assume that $C S I_{k}$ represents an indication of the maximum MCS level supported by the terminal in order to successfully decode the received signal with a Bit Error Rate (BER) smaller than a pre-defined target value. Let $\mathcal{U}_{m}=\left\{k \in \mathcal{K} \mid C S I_{k} \geq\right.$ $m\}$ denote the set of users belonging to $\mathcal{K}$ which successfully support the $m$-th MCS level, with $m=1, \ldots, M$.

According to the CMS policy, the multicast transmission is performed at the data rate allowed by the user in the worst channel conditions. In this case, all the $R$ resources are scheduled with a MCS equal to $m=\min _{k \in \mathcal{K}} C S I_{k}$. As a consequence, all the multicast group members are served with the same data rate determined by the users located at the cell border which, on average, experience bad reception conditions.

With a subgrouping scheme, the multicast user set and the available frequency resources are split into $S$ subgroups. Each subgroup contains the users experiencing CSI values in a given range, which is non overlapping with the range of another subgroup. Each subgroup is then characterized by a different MCS, and all the users in a given subgroup are served with the MCS associated to the minimum CSI feedback among those received from the users belonging to the subgroup. Under this assumption, the number of subgroups $S$ varies from 1 to $M$. Logically, the CMS policy can be seen as a particular case of subgroup-based resource allocation with only a single group activated $(S=1)$.

\section{B. Subgroup-based Resource Allocation}

The base station collects the CSI feedbacks from each multicast group member. Accordingly, the station creates the $\mathcal{K}$ and $\mathcal{U}_{m}$ (with $m=1, \ldots, M$ ) sets. Based on the collected CSI information, the RRM policy determines the best subgroup formation scheme. Each formation is denoted by $(i)$ the number $S$ of subgroups to enable, (ii) the MCS for each subgroup, and (iii) the amount of resources associated to each subgroup. We indicate a subgroup configuration with $\mathcal{R}=\left\{r_{1}, r_{2}, \ldots, r_{M}\right\}$, where $0 \leq r_{m} \leq R$. If $r_{m} \in \mathcal{R}$ is greater than zero, then the subgroup related to the $m$-th MCS level is enabled and $r_{m}$ represents the number of resources allocated to the subgroup. If $r_{m}=0$ such a subgroup is not enabled. The number $S$ of enabled subgroups is given by the sum of items $r_{m} \in \mathcal{R}$ greater than zero. We denote with $d^{\text {min }}$ the minimum data rate required by the multicast service.

The best formation scheme is the one that maximize a given cost function. In this paper we consider two different target cost functions.
1) Maximum Throughput: One of the main concerns related to the conventional multicast allocation is the poor throughput performance due to the presence of cell-edge users. With the aim to overcome this limit, a resource allocation can be adopted for multicast subgroup formation that aims to maximize the Aggregate Data Rate, as also considered in [13] [22]. Hence, the subgrouping resource allocation problem can be expressed as follows:

$$
\begin{array}{ll} 
& \underset{\mathcal{R}}{\arg \max } \sum_{m=1}^{M} c_{m} B_{o} r_{m}\left|\mathcal{U}_{m}\right| \\
\text { s.t. } & \sum_{m=1}^{M} r_{m}=R \\
& r_{m}>0, \text { with } m=\min _{k \in \mathcal{K}} C S I_{k} \\
& c_{m} B_{o} r_{m} \geq d^{m i n}, \forall r_{m} \in \mathcal{R} \mid r_{m}>0
\end{array}
$$

where $c_{m} B_{o} r_{m}$ represents the data rate assigned to an enabled subgroup, which depends on the MCS level (i.e., $c_{m}$ ) and the number of assigned frequency resources (i.e., $r_{m}$ ). Constraint (2a) shows that all the available resources are exploited by the resource allocation algorithm. Constraint ( $2 b)$ guarantees that all multicast destinations are served by enabling a subgroup with the MCS supported by the worst condition user. Finally, constraint (2c) indicates that each enabled subgroup must be served with at least the minimum data rate required by the multicast service.

In summary, this approach exploits the potentialities of OFDMA by performing subgroup formation based on the heterogeneous channel conditions experienced by the user terminals. According to eq. (1), terminals with good channel quality will receive higher data rate as they will be served in subgroups with higher MCS levels, and the influence of edge-cell users in terms of system performance degradation is reduced. It is worth noting that, like with the CMS policy, maximizing the ADR in the considered subgrouping approach allows to maximize the multicast gain, by serving the whole set of destinations involved in the multicast session.

2) Proportional Fairness: Although the ADR maximization can be considered as an efficient strategy from a provider point of view, because it allows guaranteeing the highest achievable system throughput, it does not consider the fairness among users. Authors in [26] proved that proportional fairness in the resource allocation can be obtained by maximizing the sum of the logarithm of the data rate. Thus, the subgrouping problem can be properly expressed as follows:

$$
\underset{\mathcal{R}}{\arg \max } \sum_{m=1}^{M} \log \left(c_{m} B_{o} r_{m}\right)\left|\mathcal{U}_{m}\right|
$$

subject to the same constraints in (2a), (2b), and (2c). According to eq. (3), the inter-subgroup fairness is guaranteed and the multi-user diversity is successfully exploited because terminals with good channel quality will be served with higher data rates. 
C. Solving the optimization problem through exhaustive search

The optimization problems defined in (1) and (3) can be solved through an Exhaustive Search Scheme (ESS). The ESS approach selects, among a set of admissible solutions, the one which maximizes the target cost function. In this case the search space is composed by all the feasible subgroup configurations to be assumed by $\mathcal{R}$. With $M$ potential subgroups to enable and $R$ resources to share among such subgroups, the computational complexity related to the ESS policy is given by $\mathcal{O}\left(M^{R}\right)$ [13]. Although the ESS can find the subgroup configuration that guarantees the highest cost function value, the number of feasible solutions exponentially increases with the number of available frequency resources. This high complexity cost makes the ESS an un-suitable policy in practical systems, where the resource allocation has to be performed within a scheduling frame lasting no more than $10 \mathrm{~ms}$ [14]. As a consequence, low-complexity near-optimal algorithms are needed to reduce the time required for RRM allocation procedures. This key challenge will be the focus of the next Section, which describes the proposed novel scheme for subgroup formation in OFDMA systems.

\section{The Frequency DOMAin SubGRoup AlgoriThM}

We propose the Frequency domAin Subgroup algoriThm (FAST) to provide a near-optimal solution close to the one obtained by the ESS while considerably reducing the computational cost of multicast subgrouping. Every scheduling frame, FAST finds the best subgroup configuration according to the CSI feedbacks collected by the base station. The formed subgroups, and the related resources assigned to them, may dynamically change frame by frame to adapt to the variations of user channel conditions.

FAST (summarized in Table I) is an iterative algorithm based on a greedy approach. At every iteration, FAST increases the number of enabled subgroups and searches the most suitable subgroup configuration that allows the target cost function to be higher than in the previous iteration. Iterations terminate when no further improvements in terms of objective function are achieved. As mentioned in Section III-B, different goals in the subgroup creation can be achieved by properly adapting the target cost function. We indicate with $\Omega$ the target cost function exploited in FAST. In case of ADR maximization, this function is equal to $\Omega^{M T}=\sum_{m=1}^{M} c_{m} B_{o} r_{m}\left|\mathcal{U}_{m}\right|$, while it is equal to $\Omega^{P F}=\sum_{m=1}^{M} \log \left(c_{m} B_{o} r_{m}\right)\left|\mathcal{U}_{m}\right|$ in case of a proportional fairness allocation.

FAST exploits two different sets. The first set is denoted with $\mathcal{M}$ and contains the feasible MCSs, i.e., the MCSs supported by at least one terminal. As mentioned above, each subgroup is related to a different MCS. Hence, the $\mathcal{M}$ set collects the MCSs to be evaluated for subgroup formation. Logically, the maximum admissible number of subgroups coincides with the cardinality of this set, i.e., $|\mathcal{M}|$. The second set used by FAST is indicated with $\mathcal{M}_{E}$ and represents the set of enabled MCSs. During the first iteration $(t=1)$, FAST performs the CMS resource allocation by scheduling all the $R$ frequency resources with the MCS supported by the user with the worst channel conditions; this configuration is indicated with $\tilde{\mathcal{R}}_{1}$. Once this step is performed, FAST stores the enabled MCS in the $\mathcal{M}_{E}$ set. Finally, FAST calculates the cost function value, denoted with $\tilde{\Omega}_{1}$, related to the enabled configuration.

At the second iteration $(t=2)$, FAST evaluates if there exists a subgroup configuration formed by two subgroups, which allows to increase the objective function value compared to the previous iteration. Each candidate configuration evaluated during this step is denoted with $\mathcal{R}_{j, 2}$; it considers the case when another subgroup, associated to the $j$-th MCS level, is enabled in addition to the subgroup enabled at the first iteration. In each configuration the new enabled subgroup will be related to a MCS among the feasible ones not already enabled. This means that $j \in \mathcal{M} \backslash \mathcal{M}_{E}$. As a consequence, in this step $|\mathcal{M}|-1$ configurations are examined. Among all candidate solutions, FAST selects the configuration $\mathcal{R}_{j^{*}, 2}$ which guarantees the highest target cost function value, denoted with $\tilde{\Omega}_{2}$. If $\tilde{\Omega}_{2}>\tilde{\Omega}_{1}$, then the target subgroup configuration formed by two subgroups is selected by FAST as input for the next iteration, and it will be denoted with $\tilde{\mathcal{R}}_{2}$. Accordingly, the set of enabled MCSs, i.e., $\mathcal{M}_{E}$, is updated. If $\tilde{\Omega}_{2} \leq \tilde{\Omega}_{1}$, FAST stops and $\tilde{\Omega}_{1}$ is the output of the algorithm.

At the generic $t$-th iteration, all the combinations made up of $t$ subgroups, including those picked at the previous iterations, are examined. The overall number of combinations created will be equal to $|\mathcal{M}|-t+1$. The algorithm is iterated until either no further cost function improvement is achieved or all the admissible subgroups have been chosen, i.e., $t=|\mathcal{M}|$.

A key issue is related to the distribution of the available frequency resources among the enabled subgroups. Approaches such as random resource distribution introduce several inefficiencies [13], and for this reason we define a dynamical resource distribution which takes into account, for each subgroup, the spectral efficiency and the number of served users. A candidate solution at the generic $t$-th iteration is represented by $\mathcal{R}_{j, t}$, with $\left|\mathcal{R}_{j, t}\right|=M$. As mentioned above, items $r_{m} \in \mathcal{R}_{j, t}$ greater than zero denote the enabled subgroups with the related number of assigned resources. Consequently, in each configuration $\mathcal{R}_{j, t}$, with $j \in \mathcal{M} \backslash \mathcal{M}_{E}$, we have that $r_{m}>0 \forall m \in \mathcal{M}_{E} \cup\{j\}$, whereas other items $r_{m}$ are set to zero. In order to achieve the value of $r_{m}$ for each subgroup, we define a weight [15], $\alpha_{j, m}$, which takes into account the spectral efficiency and the number of served users of the subgroup:

$$
\alpha_{j, m}= \begin{cases}\frac{c_{m} B_{o}\left|\mathcal{U}_{m}\right|}{\sum_{n \in \mathcal{M}_{E} \cup\{j\}} c_{n} B_{o}\left|\mathcal{U}_{n}\right|}, & \forall m \in \mathcal{M}_{E} \cup\{j\} \\ 0, & \text { otherwise }\end{cases}
$$

Hence, the value of each item $r_{m} \in \mathcal{R}_{j, t}$ is equal to:

$$
r_{m}= \begin{cases}1+\left\lfloor\alpha_{j, m} \cdot\left(R^{*}\right)\right\rfloor, & \text { if } \alpha_{j, m}>0 \\ 0, & \text { otherwise }\end{cases}
$$

The higher the spectral efficiency and the number of destinations related to a subgroup, the greater the number of assigned resources. In Eq. (5), the value $R^{*}$ represents the number of available resources after the allocation of the minimum required rate to each subgroup in the candidate configuration. 
Hence, (5) guarantees the minimum rate requirements to each enabled subgroup in order to satisfy the constraint (2c). The floor function does not guarantee that all the resources are exploited (the number of not assigned resources is bounded by $t-1)$. The remaining resources are assigned through a roundrobin scheme, from the subgroup with the highest weight $\alpha_{j, m}$ to the subgroup with the lowest one. It is worth noting that, according to (4) and (5), the resource distribution at every iteration varies for each candidate configuration.

TABLE I

THE FAST APPROACH

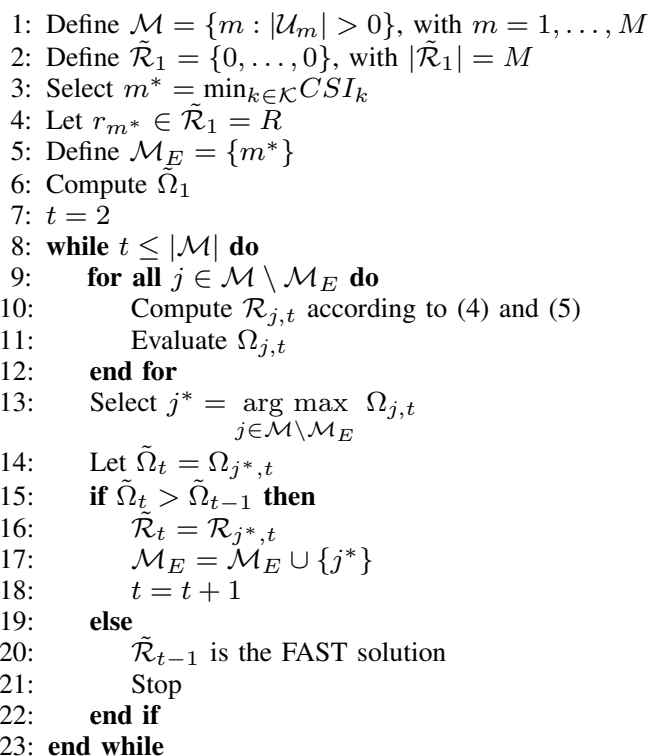

From Table I, FAST has an overall complexity equal to $\mathcal{O}\left(M^{3}\right)$, where $M$ is the maximum number of subgroups to enable. Therefore, FAST is more suitable than ESS for real implementations. It is worth underlining that the computational cost of the proposed near-optimal algorithm does not depend on the number of frequency resources reserved for the multicast service and the number of multicast destinations. Finally, it is worth noticing that the behavior of the proposed FAST is not influenced by the cost function selected for subgroup formation. Hence, by properly tuning the cost function $\Omega$, the proposed subgroup-based RRM framework can be easily extended to support different subgroup formation policies.

\section{Simulation Model and Results}

\section{A. Simulation Model}

To demonstrate the effectiveness of the proposed subgrouping approaches in OFDMA-based systems, simulations are carried out by considering the LTE radio mobile system. Such a system guarantees low latency, increased system capacity, and improved spectral efficiency [1]. Moreover, since it is designed to efficiently work with the MBMS (Multimedia Broadcast Multicast Service) standard [16], LTE allows optimized multicast transmissions in both the core and radio access network. This aspect probably makes LTE the most promising wireless system able to support high-quality group-oriented services.
The RRM procedures in LTE are performed by the LTE scheduler, designed to efficiently handle resource allocation in the time and frequency domains [17]. In the frequency domain, LTE manages the spectrum in terms of sub-channels of $180 \mathrm{kHz}$ named Resource Blocks (RBs). In the time domain, the available resources are assigned by the LTE base station every Transmission Time Interval (TTI), lasting $1 \mathrm{~ms}$. In order to perform the link adaptation, LTE defines the Channel Quality Indicator (CQI) feedback which represents the maximum MCS supported by a terminal according to the experienced SINR value. Transmission parameters are adapted every CQI Feedback Cycle (CFC) according to the CQI values collected by the base station in order to fulfill channel quality variations. Table II lists the CQI levels for the LTE system, the related MCSs with their respective spectral efficiency values. According to Table II, LTE has $M=15$ different MCSs to exploit for subgroup creation.

TABLE II

CQI-MCS MAPPING IN LTE [18]

\begin{tabular}{cccc}
\hline $\begin{array}{c}\text { CQI } \\
\text { index }\end{array}$ & $\begin{array}{c}\text { Modulation } \\
\text { Scheme }\end{array}$ & Code rate & $\begin{array}{c}\text { Spectral Efficiency } \\
{[\mathrm{bit} / \mathrm{s} / \mathrm{Hz}]}\end{array}$ \\
\hline 1 & QPSK & 0.076 & 0.1523 \\
2 & QPSK & 0.120 & 0.2344 \\
3 & QPSK & 0.190 & 0.3770 \\
4 & QPSK & 0.300 & 0.6016 \\
5 & QPSK & 0.440 & 0.8770 \\
6 & QPSK & 0.590 & 1.1758 \\
7 & 16-QAM & 0.370 & 1.4766 \\
8 & 16-QAM & 0.480 & 1.9141 \\
9 & 16-QAM & 0.600 & 2.4063 \\
10 & 64-QAM & 0.450 & 2.7305 \\
11 & 64-QAM & 0.550 & 3.3223 \\
12 & 64-QAM & 0.650 & 3.9023 \\
13 & 64-QAM & 0.750 & 4.5234 \\
14 & 64-QAM & 0.850 & 5.1152 \\
15 & 64-QAM & 0.930 & 5.5547 \\
\hline
\end{tabular}

The performance evaluation presented in this work is based on the guidelines defined in [19]. Channel quality for each terminal is evaluated in terms of the SINR measured over each sub-carrier [20]:

$$
S I N R_{i}=\frac{P_{0} \times P L_{0} \times h_{0, i}}{\sum_{j=1}^{N_{B S}}\left(P_{j} \times P L_{j} \times h_{j, i}\right)+N_{o}}
$$

where $P_{0}$ is the transmission power, $P L_{0}$ the path loss, and $h_{0}$ the small scale fast fading of the link between the terminal and the serving base station; $P_{j}, P L_{j}$ and $h_{j}$ are respectively the transmission power, the path loss plus shadow fading, and the small scale fast fading of the link between the terminal and the $j$-th interfering base station. Finally, $N_{o}$ is the noise power. These values are mapped into the effective SINR according to the Exponential Effective SIR Mapping (EESM) [20]:

$$
S I N R_{e f f}=-\beta \ln \left(\frac{1}{N_{\text {sub }}} \sum_{i=1}^{N_{\text {sub }}} e^{-\frac{S I N R_{i}}{\beta}}\right)
$$

where $N_{s u b}$ is the total number of sub-carriers. The parameter $\beta$ is a scaling factor used to dynamically adjust, every scheduling frame, the mismatch between the actual and the predicted BLock Error Rate (BLER). We modeled the value $\beta$ according to [21]. Finally, SINR is mapped to a CQI level (i.e., MCS) 
which ensures a BLER target value smaller than 10\% [20]. Main simulation assumptions are listed in Table III. Outputs are achieved by averaging a sufficient number of simulation results to obtain $95 \%$ confidence intervals.

TABLE III

Main Simulation PARAmeters

\begin{tabular}{ll}
\hline Parameter & Value \\
\hline Distance attenuation & $128.1+37.6^{*} \log (\mathrm{d}), \mathrm{d}[\mathrm{km}]$ \\
Shadow fading & Log-normal,0 mean, $\sigma=8[\mathrm{~dB}]$ \\
Shadowing Correlation dis- & $50 \mathrm{~m}[19]$ \\
tance & \\
Fast Fading & ITU-R PedB (extended for OFDM) \\
Carrier frequency & $2 \mathrm{GHz}$ \\
Cell layout & $3 \mathrm{GPP}$ Macro-cell case \#1, Hexagonal \\
& grid, 19 cell sites, 3 sectors per site [19] \\
Inter Site Distance & $500 \mathrm{~m}$ \\
RB size & $12 \mathrm{sub}-\mathrm{carriers}, 0.5 \mathrm{~ms}$ \\
Sub-carrier spacing & $15 \mathrm{kHz}$ \\
Data/Control OFDM sym- & $11 / 3$ \\
bols & \\
CQI scheme & Wideband \\
BLER target & $10 \%$ \\
TTI & $1 \mathrm{~ms}$ \\
CQI Feedback cycle & $10 \mathrm{~ms}$ \\
Antenna pattern & {$[25]$} \\
EUTRA UE & Antenna gain 0 dBi, Noise Figure 9 dB \\
EUTRA Node-B & {$[19]$} \\
eNodeB transmit power & Antenna gain 14 dBi, Noise Figure 5 \\
MIMO Configuration & dB [19] \\
Thermal Noise & $13 \mathrm{dBm}$ [19] $2 \mathrm{Rx}$ \\
QoS minimum requirement & $100 \mathrm{kbps}$ \\
\hline
\end{tabular}

In order to assess the effectiveness of the subgroup-based RRM policies (both Maximum Throughput and Proportional Fairness), we compare them with the CMS algorithm, which represents the standard solution for multicast traffic delivery in MBMS. To assess the near-optimal behavior of the proposed FAST for subgroup formation, we compare its performance against the ESS used as a benchmark and two low-complexity schemes, i.e., SMS [13] and MGGA [22], mentioned in the related work section.

We consider two scenarios with stationary user distributions: (i) Uniform Scenario, where the users are uniformly distributed within the whole cell coverage area, see Fig. 2(a); (ii) Sparse Scenario, which represents a typical on-campus scenario where the users distributed over different concentrated areas as shown in Fig. 2(b), experience heterogeneous channel conditions.

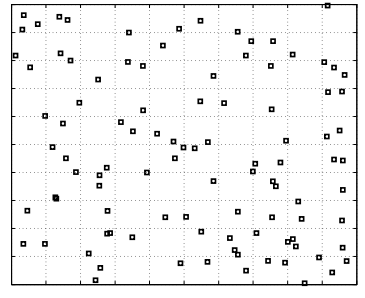

(a)

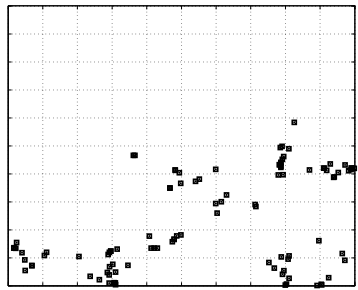

(b)
Fig. 2. Uniform (a) and Sparse (b) user distribution within the cell.

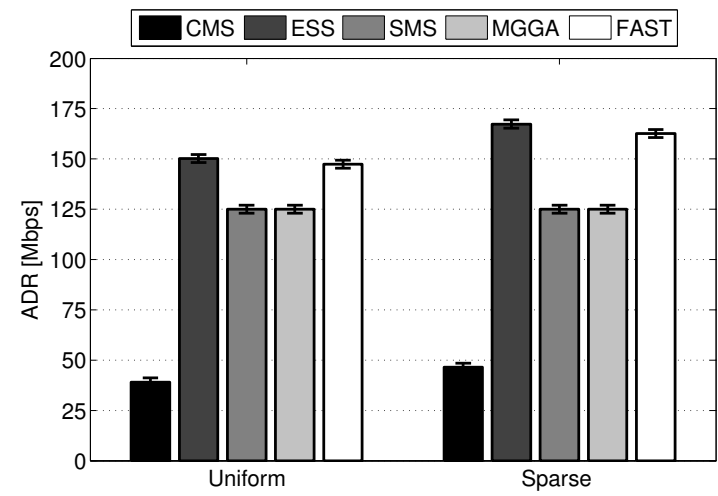

(a)

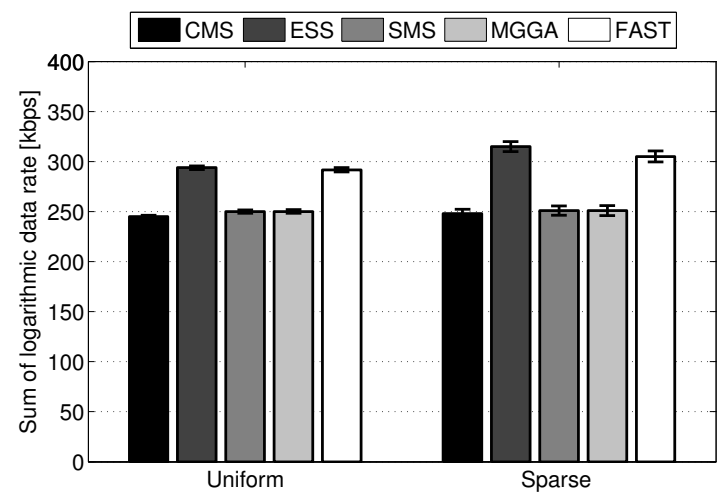

(b)

Fig. 3. Cost function performance for maximum throughput (a) and proportional fairness (b) allocation.

\section{B. Simulation Results}

The first simulation (Fig. 3) is aimed at demonstrating the near-optimal performance achieved by the FAST policy when applied with the Maximum Throughput and the Proportional Fairness allocation schemes. We assume that $K=100$ users join the multicast group. We consider a channel bandwidth equal to $3 \mathrm{MHz}$, i.e., $R=15$ frequency resources (RBs) are available for the multicast session. In Fig. 3(a) the achieved ADR is shown when the subgroup forming policy aims to maximize the throughput through different techniques (ESS, SMS, MGGA). CMS is shown for comparison with the groupbased solutions. As expected, CMS reaches the lowest ADR value, $42.8 \mathrm{Mbps}$ on average due to the cell-edge users. A meaningful improvement is attained by all subgroup-based algorithms; specifically the ESS technique offers the maximum ADR equal to $159.5 \mathrm{Mbps}$, on average. These results demonstrate $(i)$ how much the CSM policy is strongly affected by cell-edge users with poor channel conditions which bound the data rate performance of the overall multicast group, and (ii) how much the subgroup-based approach introduces significant gains in terms of system performance with a consequent better radio channel exploitation. By analyzing the results achieved by the low-complexity algorithms, both SMS and MGGA 
reach an $\mathrm{ADR}$ value equal to $125 \mathrm{Mbps}$, on average, in all considered scenarios. Finally FAST guarantees an ADR value equal to $155 \mathrm{Mbps}$, which is very close to the result achieved through an exhaustive search. It clearly appears that FAST offers a better near-optimal performance compared to SMS and MGGA. Indeed, the mismatch in terms of ADR, i.e., $\Delta_{A D R}$, between the ESS and the SMS algorithm is equal to $16 \%$ in the Uniform scenario and to $33 \%$ in the Sparse scenario, and almost the same values hold for MGGA. The $\Delta_{A D R}$ of FAST is always equal to $3 \%$ in both addressed scenarios. Moreover, it is worth underlining that subgrouping in the Sparse scenario achieves higher ADR than in the Uniform scenario, thanks to the multi-user diversity exploitation. This analysis also demonstrates that the close-to-optimum performance of FAST is not influenced by the user distribution within the cell. Indeed, while the $\Delta_{A D R}$ of SMS and MGGA is higher in the Sparse scenario, the $\Delta_{A D R}$ of FAST is equal in both addressed environments. The analysis for the proportional fairness allocation is shown in Fig. 3(b). In this case, we consider the performance in terms of sum of logarithmic data rate since this is the target metric for this strategy. From the achieved simulation results, we can note that the performance of ESS is equal to $304 \mathrm{kbps}$, on average, while this value is equal to 250 kbps, on average, for both SMS and MGGA. The proposed FAST achieved a performance equal to $298 \mathrm{kbps}$, on average. Again, the performance closest to optimum is obtained by FAST, that has a mismatch compared to the optimal value, i.e., $\Delta_{P F}$, equal to $3 \mathrm{~A}$ further comparison of CMS, ESS, SMS, MGGA and FAST policies can be found in Tables IV and $\mathrm{V}$ which list the parameter values related to a sample simulation in a Sparse scenario for the Maximum Throughput and the Proportional Fairness allocation schemes, respectively. The ESS and the FAST algorithms enable a "similar" subgroup configuration with two subgroups with the same MCSs. In both considered cases, the non-optimal performance of FAST is only related to the different resource distribution among the enabled subgroups with a consequent difference in terms of subgroup data rates. Instead, the output of SMS and MGGA is a subgroup configuration composed of 3 subgroups in case of ADR maximization, and this involves a significant difference in terms of data rate experienced by multicast users compared to the ESS case. In the proportional fairness case, SMS and MGGA enable two subgroups, but with different MCSs and resource distribution with respect to ESS.

This near-optimal behavior of FAST can be fully explored by evaluating the empirical cumulative distribution function of the data rates achieved by the users, i.e., the Network Coverage. In particular, in Fig. 4 is shown the Network Coverage for both Uniform and Sparse scenarios in the case of a maximum throughput allocation. We can note that the high mismatch of SMS and MGGA compared to ESS is highlighted from the different behavior in terms of Network Coverage, whereas the FAST has a behavior very close to that of ESS in both the addressed user distribution scenarios .

Another important aspect needs to be stressed. From Table IV, FAST enables two subgroups, While SMS and MGGA enable a configuration composed by three subgroups. This means that up to three iterations are required for the FAST
TABLE IV

COMPARISON OF CMS AND SUBGROUPING APPROACHES FOR MAXIMUM THROUGHPUT ALLOCATION

\begin{tabular}{|c|c|c|c|c|c|}
\hline & $\begin{array}{l}\text { Number of } \\
\text { Subgroups }\end{array}$ & $\begin{array}{l}\text { MCS } \\
\text { Index }\end{array}$ & $\begin{array}{l}\text { Number } \\
\text { of RBs }\end{array}$ & $\begin{array}{c}\text { Data Rate } \\
\text { [Mbps] }\end{array}$ & $\begin{array}{c}\text { Users } \\
{[\%]}\end{array}$ \\
\hline CMS & 1 & 1 & 15 & 0.3749 & 100 \\
\hline \multirow{2}{*}{ ESS } & \multirow{2}{*}{2} & 1 & 4 & 0.1 & 100 \\
\hline & & 8 & 11 & 3.45 & 40 \\
\hline \multirow{3}{*}{ SMS } & \multirow{3}{*}{3} & 1 & 4 & 0.1 & 100 \\
\hline & & 4 & 2 & 0.1974 & 83 \\
\hline & & 7 & 9 & 2.1803 & 19 \\
\hline \multirow{3}{*}{ MGGA } & \multirow{3}{*}{3} & 1 & 4 & 0.1 & 100 \\
\hline & & 4 & 4 & 0.3948 & 83 \\
\hline & & 7 & 7 & 1.6957 & 19 \\
\hline \multirow{2}{*}{ FAST } & \multirow{2}{*}{2} & 1 & 5 & 0.125 & 100 \\
\hline & & 8 & 10 & 3.1363 & 40 \\
\hline
\end{tabular}

TABLE V

COMPARISON OF CMS AND SUBGROUPING APPROACHES FOR PROPORTIONAL FAIRNESS ALLOCATION

\begin{tabular}{cccccc}
\hline & $\begin{array}{c}\text { Number of } \\
\text { Subgroups }\end{array}$ & $\begin{array}{c}\text { MCS } \\
\text { Index }\end{array}$ & $\begin{array}{c}\text { Number } \\
\text { of RBs }\end{array}$ & $\begin{array}{c}\text { Data Rate } \\
{[\mathrm{Mbps}]}\end{array}$ & $\begin{array}{c}\text { Users } \\
{[\%]}\end{array}$ \\
\hline \hline \multirow{2}{*}{ ESS } & 1 & 3 & 15 & 0.9278 & 100 \\
\hline \hline \multirow{2}{*}{ SMS } & \multirow{2}{*}{2} & 3 & 2 & 0.1238 & 100 \\
\cline { 3 - 6 } & \multirow{2}{*}{2} & 5 & 13 & 1.87 & 97 \\
\hline \hline \multirow{2}{*}{ MGGA } & \multirow{2}{*}{2} & 6 & 11 & 0.2474 & 100 \\
\hline \hline \multirow{2}{*}{ FAST } & \multirow{2}{*}{2} & 3 & 4 & 0.2472 & 85 \\
\cline { 3 - 6 } & & 3 & 11 & 2.72 & 100 \\
\hline & & 5 & 12 & 0.1857 & 100 \\
\hline
\end{tabular}

convergence, whereas this number increases up to 13 for SMS. By considering the MGGA, this policy requires to evaluate a large number of generations (i.e., iterations) before to achieve the final subgroup configuration. ${ }^{1}$ As a consequence, FAST needs less operations, compared to SMS and MGGA, in order to obtain the final subgroup configuration. The same analysis can be considered for the case of proportional fairness allocation, shown in Table V. In this case, all considered low complexity policies enable two subgroups. Again, FAST needs three iterations for convergence while both SMS and MGGA require a larger number of iterations before to obtain the final configuration. The different behavior of FAST, SMS and MGGA is highlighted in Fig. 5, which shows the number of operations required by the considered policies as a function of the number of subgroups enabled in the output configuration. SMS and MGGA require less operations than FAST only when the output configuration is composed of 14 or 15 subgroups. If we consider that the number of enabled subgroups is up to three (case of maximum throughput allocation in Table IV), FAST allows a reduction in terms of operations by a percentage equal to $97 \%$ and $94 \%$ with respect to SMS and MGGA, respectively.

Another simulation campaign has been conducted to asses if the very close to optimum behavior of FAST is affected by the number of multicast destinations and available scheduling resources. We focus on two scenarios: (a) a fixed channel bandwidth equal to $3 \mathrm{MHz}$ (i.e., $15 \mathrm{RBs}$ ) and a varying number

\footnotetext{
${ }^{1}$ The overall complexity of SMS and MGGA in the referenced LTE scenario is equal to $\mathcal{O}\left(M^{4}\right)$.
} 


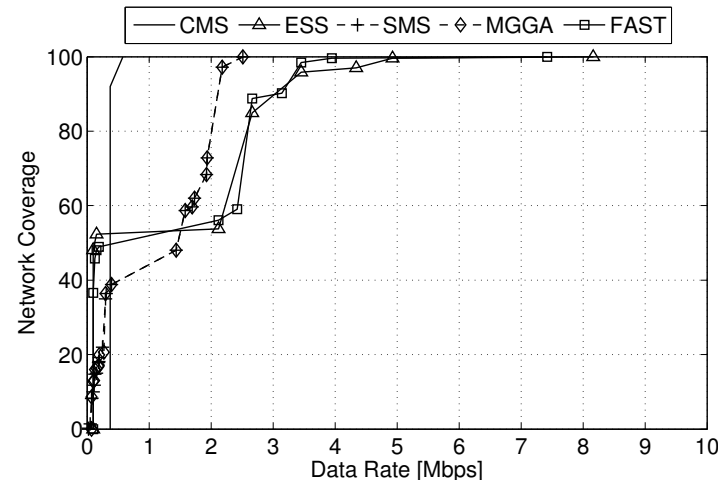

(a)

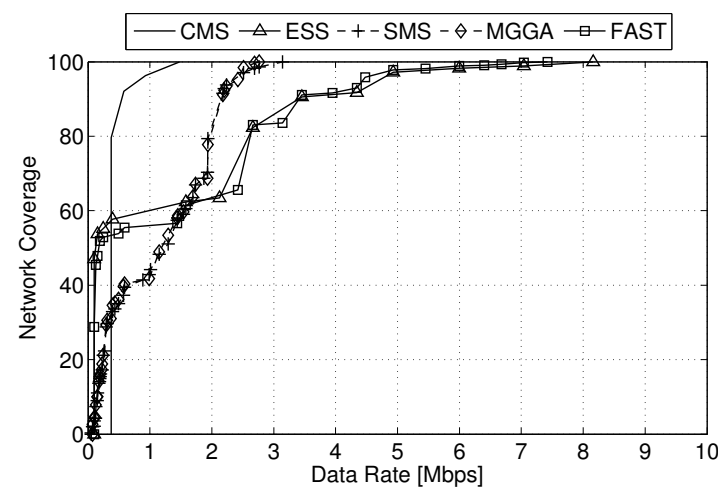

(b)

Fig. 4. Network Coverage Analysis for maximum throughput allocation in Uniform (a) and Sparse (b) scenarios.

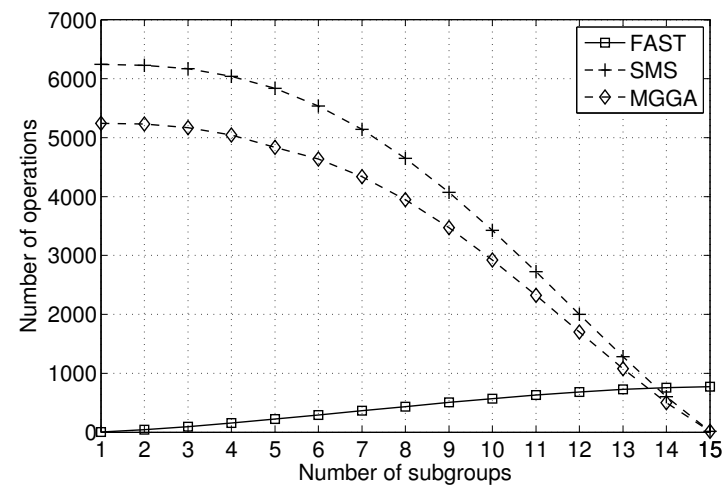

Fig. 5. Comparison of FAST, SMS, and MGGA in terms of number of operations required for convergence. of multicast receivers from 10 to $100 ;(b)$ a group of 100 users and a varying channel bandwidth from $1.4 \mathrm{MHz}$ (i.e., $6 \mathrm{RBs}$ ) up to $5 \mathrm{MHz}$ (i.e., $25 \mathrm{RBs}$ ). In both scenarios we assumed that users are distributed according to the Sparse distribution. We addressed the cases of maximum throughput and proportional fairness allocation. When focusing on the former case, shown in Figures 6 and 7, we observe that the highest mismatch, equal to 4\%, between the ADR of ESS and FAST policies is achieved when a few users (namely 10) compose the multicast group or in cell deployments with high number of resources, namely 20-25 RBs. In scenarios with large multicast groups or limited channel bandwidth the $\Delta_{A D R}$ decreases down to $0.3 \%$. By considering the proportional fairness case, shown in Figures 8 and 9, we can note that the highest mismatch, i.e., 4\%, between ESS and FAST is obtained in scenarios with small multicast groups, i.e., 10 multicast users. Again, the mismatch $\Delta_{P F}$ decreases when the number of multicast destinations increases.

Therefore, by comparing the performance of ESS, FAST, SMS and MGGA, one observes that: $(i)$ the proposed FAST approach has a lower complexity cost compared to the ESS, SMS and MGGA; (ii) FAST achieves results very close to the optimal ones, and the mismatch with respect to the optimal cost function value is less than $4 \%$ either in the case of maximum throughput or proportional fairness allocation; (iii) FAST requires less number of iterations for the convergence than SMS and MGGA; (iv) the near-optimal behavior of FAST is not affected by the user distribution within the cell.

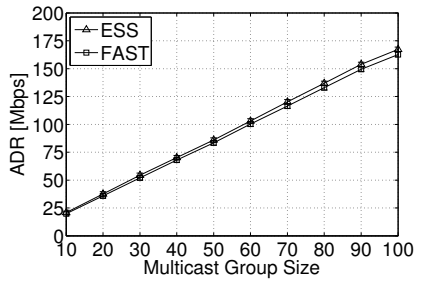

(a) $\mathrm{ADR}$

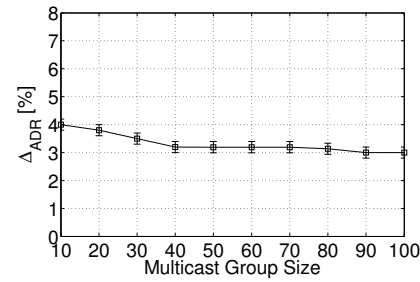

(b) ESS-FAST mismatch
Fig. 6. Performance as function of user number for the maximum throughput allocation.

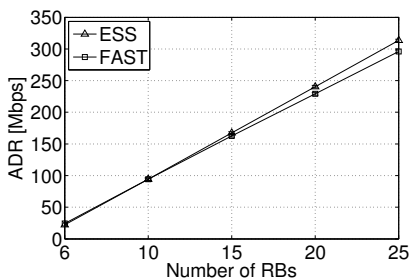

(a) ADR

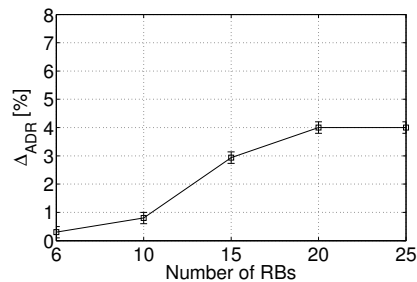

(b) ESS-FAST mismatch
Fig. 7. Performance as function of resource number for the maximum throughput allocation. 


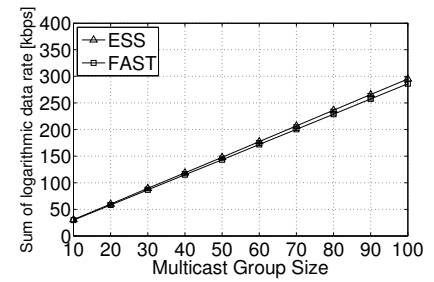

(a) Sum of logarithmic data rate

Fig. 8. Performance as function of user number for the proportional fairness allocation.

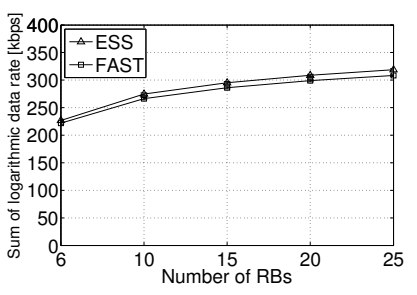

(a) Sum of logarithmic data rate

Fig. 9. Performance as function of resource number for the proportional fairness allocation.

\section{Remark on CSI Assumption}

In this work we assume that user channel conditions are represented by a single CSI value, which corresponds to the wideband CQI mode in LTE systems. To justify this assumption, in this section the impact on the proposed solution is evaluated, and it is shown that it does not introduce major errors or inefficiencies.

A generic OFDMA-based system allows to exploit the frequency selectivity by assigning to the served users the better portion of the spectrum according to their experienced channel qualities. This means that a user could potentially report a different CSI value for each available scheduling resource and that the base station could use a different MCS for each frequency resource assigned to a given data transmission. Our aim is to demonstrate if and how much the frequency selectivity affects the performance in a case of a single multicast session. For this purpose, we consider a scenario with maximum throughput allocation where we vary the number of users involved in a generic subgroup and the number of frequency resources assigned to the considered subgroup. We calculate the ADR offered by the wideband CQI mode addressed in our work and the ADR achieved by exploiting the frequency selectivity. The latter ADR value is calculated by considering that each $\mathrm{RB}$ assigned to the subgroup is served with the minimum MCS among those supported by the involved users over the considered RB. The gain in terms of ADR offered by frequency selectivity exploitation is shown in Fig. 10. It can be observed that in general the frequency selectivity exploitation does not introduce a meaningful gain in point-to-multipoint transmissions towards a single multicast group. In particular, the highest gain is of about $5 \%$ and is only obtained when the number of users in the subgroup is very low, namely 5 , and the number of assigned RBs is high, namely 100. In other cases, the introduced gain is lower or even negligible.

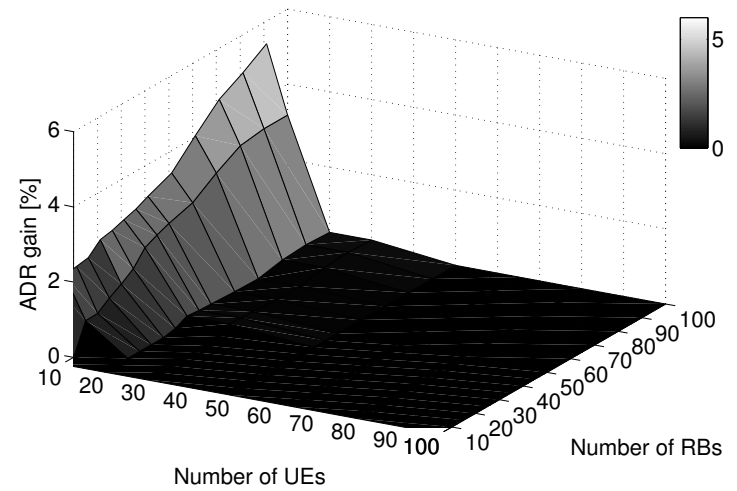

Fig. 10. Gain of frequency selectivity exploitation in a single group scenario.

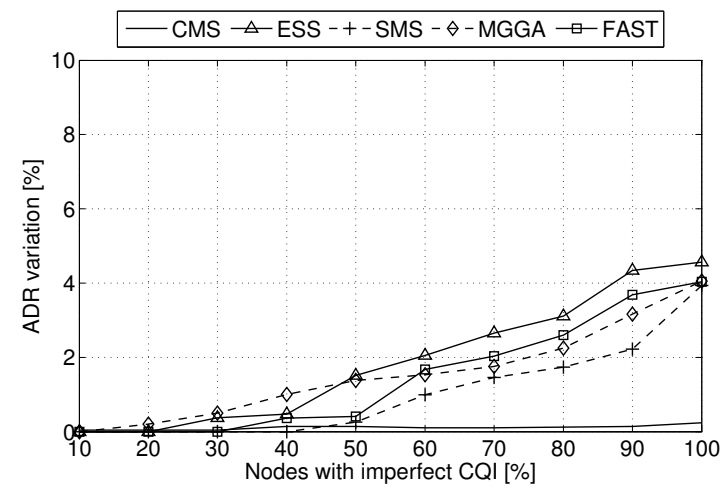

Fig. 11. ADR variation due to imperfect CQI estimation.

According to the analysis presented in this section, we can conclude that the assumption introduced in our work has a very small impact on the system results and can be, thus, considered acceptable. This is especially true if we consider that frequency selectivity involves two aspects: (i) a large amount of uplink control traffic is required for CSI feedback transmissions; (ii) the complexity of scheduling policies increases and becomes dependent from the number of users and the number of available resources. Such aspects cannot be considered negligible in case of multicast scenarios, when the number of involved users is usually high.

We also focused our attention to the impact that errors in the CSI estimation by the multicast users have on the system performances. In particular, we evaluated the robustness of addressed policies to the imperfect CSI estimation. We compared the ADR obtained when the CSI is approximated to the ideal value with the ADR achieved when the measured SINR is approximated to the ideal value and an additive independent identically distributed zero mean Gaussian error [23], such that the user experiences one level variation in the measured CQI. In Fig. 11, the ADR variation is plotted, by varying the 
percentage of users reporting an imperfect CSI value, up to the extreme case where all multicast destinations are affected by imperfect channel estimation. It can be observed that in general the considered solutions are robust to such errors. We can note that all subgroup-based policies show similar trends. In particular, we denote that in all tested cases all the solutions show an ADR variation always lower than $4.5 \%$ (this value is achieved by ESS in the worst case, i.e., all multicast members experience errors in channel estimation). This percentage decreases down to $1.38 \%$ when we consider the case of half portion of users with channel estimation errors. To conclude, we can state that an imperfect CSI estimation as has a very small impact on the results when adopting the subgrouping approach.

\section{CONCLUSION AND Future WORK}

In this paper we proposed a low complexity subgroupbased resource allocation scheme, i.e., FAST, for OFDMA multicast systems. The proposed policy, designed to cover different scheduling strategies by properly adapting the target cost function, overcomes the throughput limitations of conventional multicast schemes, while guaranteeing system capacity maximization. Simulation campaigns demonstrated that the proposed FAST algorithm (i) reduces the computational cost of subgroup creation, (ii) guarantees performance close to the one achieved by the exhaustive search scheme for both maximum throughput and proportional fairness allocations, and (iii) requires less iterations for convergence compared to existing approaches. The high performance level of the proposed solution makes it particularly interesting for the implementation in practical system (e.g., LTE) where resource must be allocated under strict time constraints.

\section{REFERENCES}

[1] 3GPP, TS 36.300, "Evolved Universal Terrestrial Radio Access (EUTRA) and Evolved Universal Terrestrial Radio Access Network (EUTRAN)," Rel. 11, September 2012.

[2] IEEE 802.16 - Standard for local and metropolitan area networks, Part 16: Air Interface for Broadband Wireless Access Systems, 2009.

[3] J. F. Monserrat, J. Calabuig, A. Fernandez-Aguilella, and D. GomezBarquero, "Joint Delivery of Unicast and E-MBMS Services in LTE Networks," IEEE Transactions on Broadcasting, vol. 58 , no. 2, pp. 157167, June 2012.

[4] A. Richard, A. Dadlani, and K. Kim, "Multicast scheduling and resource allocation algorithms for OFDMA-based systems: A survey," IEEE Communications Surveys and Tutorials, vol. 15, no.1, pp. 240-254, 2013.

[5] W. Rhee, and J. Cioffi, "Increase in capacity of multiuser OFDM systems using dynamic subchannel allocation," IEEE 51st Vehicular Technology Conference (VTC-Spring), vol. 2, pp. 1085-1089, 2000.

[6] A. Alexious, C. Bouras, V. Kokkinos, and G. Tsichritzis, "Communication cost analysis of MBSFN in LTE," IEEE 21st International Symposium on Personal Indoor and Mobile Radio Communications (PIMRC), pp. 1366-1371, September 2010.

[7] T. P. Low, M. O. Pun, Y. W. P. Hong, and C. C. J. Kuo, "Optimized opportunistic multicast scheduling (OMS) over wireless cellular networks," IEEE Transaction on Wireless Communications, vol. 9, no. 2, pp. 791801, September 2009.

[8] L. Zhang, Z. He, K. Niu, B. Zhang, and P. Skov, "Optimization of coverage and throughput in single-cell E-MBMS," IEEE 70th Vehicular Technology Conference Fall (VTC-Fall), pp. 1-5, September 2009.

[9] A. Alexious, C. Bouras, V. Kokkinos, A. Papazois, and G. Tsichritzis, "Spectral efficiency performance of MBSFN-enabled LTE networks," IEEE 6th International Conference on Wireless and Mobile Computing, Networking and Communications (WiMob), pp. 361-367, October 2010.
[10] P. K. Gopala, and H. E. Gamal, "Opportunistic multicasting," ThirtyEighth Asilomar Conference on Signals, Systems and Computers, November 2004.

[11] G. Araniti, V. Scordamaglia, M. Condoluci, A. Molinaro, and A. Iera, "Efficient Frequency Domain Packet Scheduler for Point-to-Multipoint Transmissions in LTE Networks," IEEE International Conference on Communications (ICC), pp. 5916-5920, June 2012.

[12] L. Militano, M. Condoluci, G. Araniti, and A. Iera, "Bargaining Solutions for Multicast Subgroup Formation in LTE," IEEE 76th Vehicular Technology Conference (VTC-Fall), September 2012.

[13] C. K. Tan, T. C. Chuah, and S. W. Tan, "Adaptive multicast scheme for OFDMA-based multicast wireless systems," Electronics Letters, vol. 47, no. 9, pp. 570-572, April 2011.

[14] S. Deb, S. Jaiswal, and K. Nagaraj, "Real-Time Video Multicast in WiMAX Networks," IEEE 27th Conference on Computer Communications (INFOCOM), pp. 1579-1587, April 2008.

[15] Y. Jiao, M. Ma, Q. Yu, K. Yi, and Y. Ma, "Quality of service provisioning in worldwide interoperability for microwave access networks based on cooperative game theory," IET Communications, vol. 5, no. 3, pp. 284$295,2011$.

[16] 3GPP, TS 36.440, "General aspects and principles for interfaces supporting Multimedia Broadcast Multicast Service (MBMS) within EUTRAN,” Rel. 11, September 2012.

[17] K. I. Pedersen, T. E. Kolding, F. Frederiksen, I. Z. Kovács, D. Laselva, and P. E. Mogensen, "An overview of downlink Radio Resource Management for UTRAN Long-Term Evolution," IEEE Communications Magazine, vol. 47, no. 7, pp. 86-93, July 2009.

[18] 3GPP, TS 36.213, "Evolved Universal Terrestrial Radio Access (EUTRA); Physical layer procedures," Rel. 11, September 2012.

[19] 3GPP, TR 25.814, "Physical layer aspect for evolved Universal Terrestrial Radio Access (UTRA)," Rel. 7, Oct. 2006.

[20] C. Mehlführer, M. Wrulich, J. Ikuno, B. Colom, D. Bosanska, and M. Rupp, "Simulating the Long Term Evolution physical layer," 17th European Signal Processing Conference (EUSIPCO), pp. 1471-1478, August 2009 .

[21] R. Giuliano, and F. Mazzenga, "Exponential Effective SINR Approximations for OFDM/OFDMA-Based Cellular System Planning," IEEE Transactions on Wireless Communications, vol. 8, no. 9, pp. 4434-4439, September 2009.

[22] C.K. Tan, T.C. Chuah, S.W. Tan and M.L. Sim, "Efficient clustering scheme for OFDMA-based multicast wireless systems using grouping genetic algorithm," Electronics Letters, vol. 48, no.3, pp. 184-186, Feb. 2012.

[23] K. I. Pedersen, G. Monghal, I. Z. Kovacs, T. E. Kolding, A. Pokhariyal, F. Frederiksen, and P. Mogensen, "Frequency domain scheduling for OFDMA with limited and noisy channel feedback," IEEE 66th Vehicular Technology Conference (VTC-Fall), pp. 1792-1796, Oct. 2007.

[24] T. Jiang, W. Xiang, H. H. Chen, Q. Ni, "Multicast Broadcast Services Support in OFDMA-Based WiMAX Systems," IEEE Communications Magazine, vol. 45, no. 8, pp. 78-86, Aug. 2007.

[25] 3GPP, TR 25.913, "Requirements for evolved Universal Terrestrial Radio Access (UTRA) and Universal Terrestrial Radio Access Network (UTRAN)," Rel. 9, Dec. 2009.

[26] J. Y. L. Boudec, "Rate adaptation, congestion control, and fairness: A tutorial," Tech. Rep., Tut., Ecole Polytech. Fed. Lausanne, Lausanne, Switzerland, Feb. 2005. 